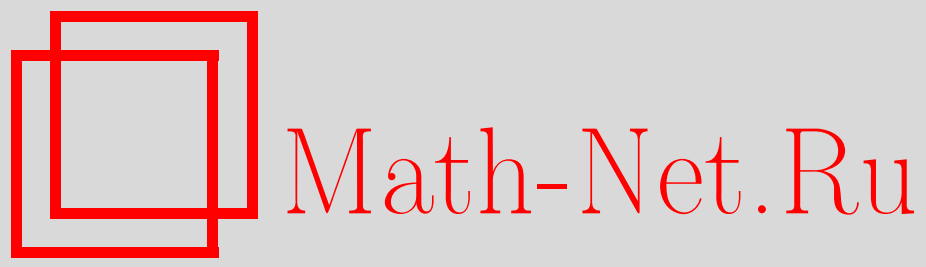

Д. В. Орлов, О двух оценках одной меры риска, Теория вероятн. и ее примен., 2008, том 53, выпуск $1,168-172$

DOI: https://doi.org/10.4213/tvp2491

Использование Общероссийского математического портала Math-Net.Ru подразумевает, что вы прочитали и согласны с пользовательским соглашением

http://www.mathnet.ru/rus/agreement

Параметры загрузки:

IP : 35.173 .219 .149

26 апреля 2023 г., 14:54:39

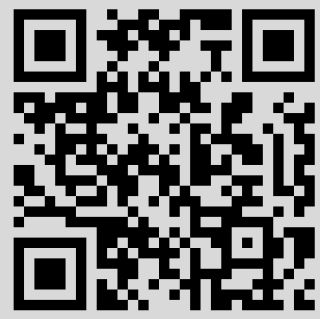


5. Manita A., Simonot F. Clustering in stochastic asynchronous algorithms for distributed simulations. - Lecture Notes in Comput. Sci., 2005, v. 3777, p. 26-37.

6. Mitra D., Mitrani I. Analysis and optimum performance of two message-passing parallel processors synchronized by rollback. - Performance Evaluation, 1987, v. 7, № 2, p. 111-124.

Поступила в редакцию 2.VI.2006

Исправленный вариант 12.XI. 2007

(C) 2008 r.

ОРЛОВ Д. В.*

\section{О ДВУХ ОЦЕНКАХ ОДНОЙ МЕРЫ РИСКА}

Изучается асимптотическое поведение двух различных эмпирических оценок когерентной меры риска (минимальный $\mathrm{V} @ \mathrm{R}$ ), а именно функционала, имеюшего вид

$$
\operatorname{MINV} @ \mathrm{R}_{\alpha}(X)=-\mathbf{E} \min \left(X_{1}, \ldots, X_{\alpha}\right),
$$

где $X_{1}, \ldots, X_{\alpha}$ - независимые копии $X$.

Ключевые слова и фразы: взвешенный V@R, когерентные меры риска, минимальный V@R, предельные теоремы для $L$-статистик.

1. Введение. Когерентные меры риска были введены в работах Ф. Артцнера, Ф. Делбаена, Ж.-М. Эбера и Д. Хиса [2], [3]. Общая когерентная мера риска - это функционал на пространстве ограниченных случайных величин (предполагается фиксированным некоторое вероятностное пространство $(\Omega, \mathscr{F}, \mathbf{P})$ ), заданный по формуле

$$
\rho(X)=-\inf _{\mathbf{Q} \in \mathscr{D}} \mathbf{E}_{\mathbf{Q}} X,
$$

где $\mathscr{D}$ - некоторое множество вероятностных мер, абсолютно непрерывных относительно $\mathbf{P}$.

Этот класс функционалов очень широк. Для его практических применений необходимо иметь более узкие, аналитически удобные подклассы. Одним из таких подклассов является взвешенный $V @ R$ (Weighted Value at Risk):

$$
\mathrm{WV} @ \mathrm{R}(X)=-\int_{\mathbf{R}} x d\left(\Psi\left(F_{X}(x)\right)\right),
$$

где $\Psi:[0,1] \rightarrow[0,1]$ - вогнутая возрастающая функция такая, что $\Psi(0)=0, \Psi(1)=1$, а $F_{X}$ обозначает функцию распределения случайной величины $X$. Эквивалентно, $\mathrm{WV} @ \mathrm{R}(X)=-\mathbf{E} Y$, где $Y$ - случайная величина с функцией распределения $F_{Y}=$ $\Psi \circ F_{X}$.

Этот класс функционалов удобен по многим причинам (см. [4], [8]). В частности, он допускает простую эмпирическую оценку. Именно, если $X$ имеет равномерное распределение на множестве $\left\{x_{1}, \ldots, x_{N}\right\}$, то, как легко видеть,

$$
\mathrm{WV} @ \mathrm{R}(X)=-\sum_{n=1}^{N} x_{(n)}\left(\Psi\left(\frac{n}{N}\right)-\Psi\left(\frac{n-1}{N}\right)\right),
$$

* Московский государственный университет им. М.В. Ломоносова, механикоматематический факультет, Ленинские горы, 119992 Москва, Россия; e-mail: orloff.dmitri@gmail.com 
где $x_{(1)}, \ldots, x_{(N)}$ 一 набор $x_{1}, \ldots, x_{N}$, упорядоченный по возрастанию. Отсюда вытекает, что для произвольной случайной величины $X$, имеющей некоторые реализации $X_{1}, \ldots, X_{N}$, ее эмпирической оценкой служит статистика

$$
\mathrm{W} \widehat{\mathrm{V} @ \mathrm{R}}(X ; N)=-\sum_{n=1}^{N} X_{(n)}\left(\Psi\left(\frac{n}{N}\right)-\Psi\left(\frac{n-1}{N}\right)\right),
$$

где $X_{(1)}, \ldots, X_{(N)}$ - порядковые статистики, построенные по выборке $X_{1}, \ldots, X_{N}$.

Kласc WV@R широк, и для практического его применения выделяют некоторых представителей (обычно это однопараметрические семейства). Одним из таких представителей является хвостовой V@R (Tail Value at Risk), определяемый как взвешенный $\mathrm{V} @ \mathrm{R}$, соответствующий $\Psi_{\lambda}(x)=\lambda^{-1} x \wedge 1$. Если случайная величина $X$ имеет непрерывное распределение, то

$$
\mathrm{TV} @ \mathrm{R}_{\lambda}(X)=-\mathbf{E}\left(X \mid X \leqslant q_{\lambda}(X)\right),
$$

где $q_{\lambda}(X)$ обозначает (правую) $\lambda$ квантиль $X$ (см. [8, раздел 4.4]).

В работе Д.Б. Мадана и А.С. Черного [5] был предложен другой интересный представитель класса WV@R, получивший название минимальный V@R и обозначаемый MINV@R. Это есть взвешенный V@R, соответствуюший $\Psi_{\alpha}(x)=1-(1-x)^{\alpha}$. Очевидно, что при $\alpha \in \mathbf{N}$

$$
\operatorname{MINV@R_{\alpha }}(X)=-\mathbf{E} \min \left(X_{1}, \ldots, X_{\alpha}\right),
$$

где $X_{1}, \ldots, X_{\alpha}$ - независимые копии $X$.

Полученное выше представление дает прозрачное и интуитивно ясное описание меры MINV@R. Но кроме того, оно дает очень простую эмпирическую оценку MINV@R. Именно, пусть $\alpha \in \mathrm{N}$ и имеются независимые реализации $X_{1}, \ldots, X_{N}$, где $N$ кратно $\alpha$. Тогда эмпирической оценкой MINV@R служит

$$
\operatorname{MINV} @ \mathrm{R}_{\alpha}^{1}(X ; N)=-\frac{\alpha}{N} \sum_{k=1}^{N / \alpha} \min \left\{X_{\alpha k+1}, \ldots, X_{\alpha(k+1)}\right\} .
$$

Но поскольку MINV@R является подклассом семейства WV@R, то его также можно оценивать при помоши описанной выше оценки WV@R, что дает оценку

$$
\mathrm{MINV} @ \mathrm{R}_{\alpha}^{2}(X ; N)=-\sum_{n=1}^{N} X_{(n)}\left(\left(\frac{N-n+1}{N}\right)^{\alpha}-\left(\frac{N-n}{N}\right)^{\alpha}\right) .
$$

Возникает вопрос: какая из оценок, (2) или (3), лучше? Этот вопрос является основным для настоящей работы. Ответом на него служит следуюшая теорема, являюшаяся нашим главным результатом. В этой теореме мы используем расширение функционала MINV@R на множество интегрируемых случайных величин, определяемое формулой (1).

Теорема 1. Пусть случайная величина $X \in L^{2}$ обладает непрерьвной функиией плотности $f(x)$. Тогда

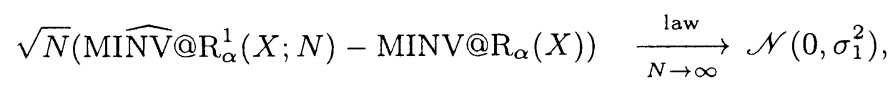

$$
\begin{aligned}
& \sqrt{N}\left(\operatorname{MINV} @ R_{\alpha}^{2}(X ; N)-\operatorname{MINV} @ R_{\alpha}(X)\right) \underset{N \rightarrow \infty}{\stackrel{\text { law }}{\longrightarrow}} \mathscr{N}\left(0, \sigma_{2}^{2}\right),
\end{aligned}
$$

где $\mathscr{N}\left(m, \sigma^{2}\right)$ обозначает нормальное распределение со средним $m$ и дисперсией $\sigma^{2}, a$

$$
\begin{aligned}
\sigma_{1}^{2} & =\alpha \mathrm{D} \min \left(X_{1}, \ldots, X_{\alpha}\right) \\
\sigma_{2}^{2} & =\int_{-\infty}^{\infty} \int_{-\infty}^{\infty} \Psi_{\alpha}^{\prime}\left(F_{X}(x)\right) \Psi_{\alpha}^{\prime}\left(F_{X}(y)\right)\left(F_{X}(\min (x, y))-F_{X}(x) F_{X}(y)\right) d x d y
\end{aligned}
$$

u $\mathbf{D}$ обозначает дисперсию. При этом $\sigma_{2}^{2}<\sigma_{1}^{2}$.

Итак, эта теорема показывает, что обе оценки (2) и (3) являются асимптотически нормальными со средним MINV@R $\mathrm{R}_{\alpha}(X)$ и асимптотическими дисперсиями порядка $N^{-1 / 2}$. Но при этом у оценки (3) асимптотическая дисперсия меньше, чем у оценки (2), т.е. оценка (3) является более точной. 
2. Доказательство теоремы 1. Для доказательства теоремы 1 нам потребуется ряд утверждений. Доказательство утверждения 1 можно найти в [7]. Доказательства утверждений 2 и 3 изложены в [9]. Доказательство утверждения 4 можно найти в [6].

Утверждение 1. Пусть $X, Y-$ две случайнье величинь, принимающие значения в $\mathbf{R}, F(x, y)=\mathbf{P}\{X \leqslant x, Y \leqslant y\}, G(x)=F(x, \infty), H(y)=F(\infty, y)$. Тогда если существуют $\mathbf{E}(X Y), \mathbf{E} X, \mathbf{E} Y$, то

$$
\operatorname{cov}(X, Y)=\int_{-\infty}^{\infty} \int_{-\infty}^{\infty}(F(x, y)-G(x) H(y)) d x d y .
$$

Пусть $\left\{X_{n}\right\}_{1}^{\infty}$ - независимые одинаково распределенные случайные величины. Для некоторой функции $J:[0,1] \rightarrow \mathbf{R}$ определим

$$
S_{N}=-\frac{1}{N} \sum_{i=1}^{N} X_{(i)} J\left(\frac{i}{N+1}\right)
$$

Определим $F_{X}^{-1}(\lambda)=\inf \left\{x: F_{X}(x)>\lambda\right\}$. Рассмотрим меру $\mu$ на $[0,1]$ такую, что $\mu((a, b])=F_{X}^{-1}(b)-F_{X}^{-1}(a)$ для любых $a, b \in[0,1], a<b$. Очевидно, что определение корректно.

Утверждение 2. Предположим, ито $\mathbf{E} X^{2}<\infty$, а тажже что $J$ ограничена и непрерывна на $[0,1] \backslash A$, где $\mu(A)=0$. Тогда

$$
\lim _{N \rightarrow \infty} N \mathbf{D} S_{N}=\sigma^{2}(J, F),
$$

гдe

$$
\sigma^{2}(J, F)=\int_{-\infty}^{\infty} \int_{-\infty}^{\infty} J\left(F_{X}(x)\right) J\left(F_{X}(y)\right)\left(F_{X}(\min (x, y))-F_{X}(x) F_{X}(y)\right) d x d y
$$

Утверждение 3. Предположим, ито $\mathbf{E} X^{2}<\infty$ и что $J$ ограничена и непрерывна на $[0,1] \backslash A$, где $\mu(A)=0$. Тогда если $\sigma^{2}(J, F)>0$, то

$$
\frac{S_{N}-\mathbf{E} S_{N}}{\mathrm{D} S_{N}} \underset{N \rightarrow \infty}{\stackrel{\text { law }}{\longrightarrow}} \mathscr{N}(0,1) .
$$

Утверждение 4. Предположим, что $X$ обладает непрерьвной плотностью $f$ (т.е. $\left.F_{X} \in C^{1}(\mathbf{R})\right)$ u $\mathbf{E} X^{2}<\infty$. Предположим также, что $J$ ограничена и имеет по меньшей мере четыре производных, которые также ограничены на $(0,1)$. Тогда

$$
\mathrm{E} S_{N}=\int_{0}^{1} F_{X}^{-1}(u) J(u) d u+O\left(\frac{1}{N}\right) .
$$

Д ок а з а т ель с т в о те о ре мы 1. Шаг 1. Докажем справедливость (4) и (6). Пусть $X_{1}, \ldots, X_{\alpha}$ - независимые копии $X$. Рассмотрим последовательность $\xi_{1}, \xi_{2}, \ldots$ независимых одинаково распределенных случайных величин таких, что $\xi_{1} \stackrel{\text { law }}{=}-\min \left(X_{1}, \ldots, X_{\alpha}\right)$. Тогда согласно центральной предельной теореме

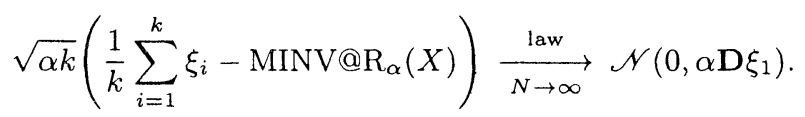

Первый шаг доказательства завершен.

Шаг 2. Докажем справедливость (5) и (7). Для функции $J(u)=\Psi^{\prime}(u)$ рассмотрим случайную величину $S_{N}$, определенную в (8). Тогда

$$
\begin{aligned}
\mathrm{E}\left(\mathrm{MINV} @ \mathrm{R}_{\alpha}^{2}(X ; N)-S_{N}\right)^{2} & =\mathrm{E}\left(\sum_{i=1}^{N} X_{(i)}\left(\int_{(i-1) / N}^{i / N} \Psi_{\alpha}^{\prime}(x) d x-\frac{1}{N} \Psi_{\alpha}^{\prime}\left(\frac{i}{N+1}\right)\right)\right)^{2} \\
& \leqslant \mathrm{E}\left(\sum_{i=1}^{N}\left|X_{(i)}\right| \frac{C_{1}}{N^{2}}\right)^{2} \leqslant C_{2} \frac{\mathrm{E} X^{2}}{N^{2}} .
\end{aligned}
$$


Отсюда, в частности, следует, что $\sqrt{N}\left(\mathrm{MINV} @ \mathrm{R}_{\alpha}^{2}(X)-S_{N}\right) \stackrel{\text { law }}{\longrightarrow} 0$ при $N \rightarrow \infty$. В силу утверждения 4 и определения MINV@R $\mathrm{R}_{\alpha}$ получаем, что

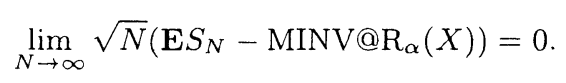

Из результатов утверждений 2 и 3 следует, что $\sqrt{N}\left(S_{N}-\mathrm{E} S_{N}\right) \stackrel{\text { law }}{\longrightarrow} \mathscr{N}\left(0, \sigma^{2}\left(\Psi_{\alpha}^{\prime}, F_{X}\right)\right)$ при $N \rightarrow \infty$. Следовательно,

$$
\begin{aligned}
& \sqrt{N}\left(\operatorname{MINV} @ R_{\alpha}^{2}(X ; N)-\operatorname{MINV} @ \mathrm{R}_{\alpha}(X)\right)=\sqrt{N}\left(\operatorname{MINV} @ \mathrm{R}_{\alpha}^{2}(X ; N)-S_{N}\right)
\end{aligned}
$$

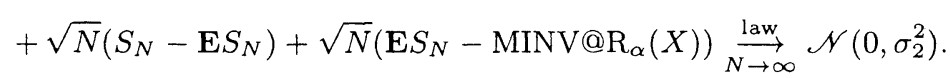

Шаг 3. Докажем, что $\sigma_{2}^{2}<\sigma_{1}^{2}$. Для случайной величины $\xi_{1}$ из первого шага доказательства верно

$$
\mathbf{D}\left(\operatorname{MINV} @ \mathrm{R}_{\alpha}^{1}(X ; N)\right)=\frac{\alpha}{N} \mathbf{D} \xi_{1} .
$$

Поскольку $X$ не имеет атомов, то $\mathbf{P}\left\{\xi_{1} \leqslant x\right\}=1-\left(1-F_{X}(x)\right)^{\alpha}$. Тогда, применяя утверждение 1 к паре $\left(\xi_{1}, \xi_{1}\right)$, получим

$$
\begin{aligned}
\alpha \mathbf{D} \xi_{1}=\alpha \int_{-\infty}^{\infty} \int_{-\infty}^{\infty}\left(1-\left(1-F_{X}(\min (x, y))\right)^{\alpha}\right. & \\
& \left.-\left(1-\left(1-F_{X}(x)\right)^{\alpha}\right)\left(1-\left(1-F_{X}(x)\right)^{\alpha}\right)\right) d x d y \\
=\alpha \int_{-\infty}^{\infty} \int_{-\infty}^{y}\left(1-F_{X}(y)\right)^{\alpha}\left(1-\left(1-F_{X}(x)\right)^{\alpha}\right) d x d y & \\
& +\alpha \int_{-\infty}^{\infty} \int_{y}^{\infty}\left(1-F_{X}(x)\right)^{\alpha}\left(1-\left(1-F_{X}(y)\right)^{\alpha}\right) d x d y
\end{aligned}
$$

С другой стороны, учитывая (9), находим, что

$$
\begin{aligned}
\sigma^{2}\left(\Psi_{\alpha}^{\prime}, F_{X}\right)= & \int_{-\infty}^{\infty} \int_{-\infty}^{y} \alpha^{2} F_{X}(x)\left(1-F_{X}(x)\right)^{\alpha-1}\left(1-F_{X}(y)\right)^{\alpha} d x d y \\
& +\int_{-\infty}^{\infty} \int_{y}^{\infty} \alpha^{2} F_{X}(y)\left(1-F_{X}(y)\right)^{\alpha-1}\left(1-F_{X}(x)\right)^{\alpha} d x d y
\end{aligned}
$$

Поскольку для любого $x$ такого, что $F_{X}(x)>0$, имеем строгое неравенство

$$
\alpha\left(1-\left(1-F_{X}(x)\right)^{\alpha}\right)>\alpha^{2} F_{X}(x)\left(1-F_{X}(x)\right)^{\alpha-1},
$$

то, в силу гладкости $F_{X}(x)$, получаем неравенство $\sigma_{2}^{2}<\sigma_{1}^{2}$.

3 а м е ч а н и е. С помошью рассуждений, проведенных на втором шаге доказательства, легко видеть, что

$$
N \mathrm{E}\left(\mathrm{MI} \widehat{\mathrm{NV}} @ \mathrm{R}_{\alpha}^{2}(X ; N)-\operatorname{MINV} @ \mathrm{R}_{\alpha}(X)\right)^{2} \longrightarrow \sigma^{2}\left(\Psi^{\prime}, F_{X}\right), \quad N \rightarrow \infty .
$$

Поскольку для любых $N$, кратных $\alpha$, имеем

$$
N \mathrm{D}\left(\mathrm{MINV} @ \mathrm{R}_{\alpha}^{1}(X)\right)=\sigma_{1}^{2},
$$

то мы видим, что оценка (3) также асимптотически лучше оценки (2) в терминах среднеквадратического отклонения.

\section{СПИСОК ЛИТЕРАТУРЫ}

1. Черныцй A.C. Нахождение справедливой цены на основе когерентных мер риска. Теория вероятн. и ее примен., 2007, т. 52, в. 3, с. 506-540.

2. Artzner P., Delbaen F., Eber J.-M., Heath D. Thinking coherently. — Risk, 1997, v. 10 , № 11, p. 68-70. 
3. Artzner P., Delbaen F., Eber J.-M., Heath D. Coherent measures of risk. - Math. Finance, 1999, v. 9, № 3, p. 203-228.

4. Cherny A.S. Weighted V@R and its properties. - Finance Stoch., 2006, v. 10, № 2, p. $367-393$.

5. Cherny A.S., Madan D. B. Coherent measurement of factor risks. http://arxiv.org: math/0605062v1

6. Jung J. On linear estimates defined by a continuous weight function. - Ark. Mat., 1956 , v. 3, p. 199-209.

7. Lehmann E. L. Some concepts of dependence. - Ann. Math. Statist., 1966, v. 37, p. $1137-1153$.

8. Föllmer H., Schied A. Stochastic Finance. An Introduction in Discrete Time. Berlin: de Gruyter, 2004, 459 p.

9. Stigler S. M. Linear functions of order statistics with smooth weight functions. - Ann. Statist., 1974, v. 2, № 4, p. 676-693; corrections: ibid., 1979, v. 7, № 2, p. 466.

Поступила в редакцию

29.VI. 2007

(c) 2008 r.

ПРЕСМАН Э. Л.*

\section{ОЦЕНКА КОНСТАНТЫ В НЕРАВЕНСТВЕ БУРКХОЛЬДЕРА ДЛЯ СУПЕРМАРТИНГАЛОВ И МАРТИНГАЛОВ ${ }^{1)}$}

Доказывается моментное неравенство типа Буркхольдера для супермартингалов и мартингалов. Доказательство сводится к усовершенствованию соответствующих рассуждений С. В. Нагаева, который получил это неравенство с бо́льшим значением константы.

Ключевые слова и фразы: математическое ожидание, мартингал, супермартингал, неравенство Буркхольдера, моменты.

1. Формулировка и обсуждение результатов. Пусть последовательность случайных величин $S_{n}=\sum_{k=0}^{n} X_{k}, n \geqslant 0$, образует супермартингал, заданный на фильтрованном вероятностном пространстве $\left(\Omega, \mathscr{F},\left(\mathscr{F}_{k}\right)_{k \geqslant 0}, \mathbf{P}\right)$, где $X_{0}=0, \mathscr{F}_{0}=$ $\{\varnothing, \Omega\}$, так что $\mathbf{E}\left\{X_{k+1} \mid \mathscr{F}_{k}\right\} \leqslant 0, k \geqslant 0$. Положим

$$
B_{n}^{2}=\sum_{k=1}^{n} \mathbf{E}\left\{X_{k}^{2} \mid \mathscr{F}_{k-1}\right\}, \quad \bar{X}_{n}=\max _{0 \leqslant k \leqslant n} X_{k}, \quad \bar{S}_{n}=\max _{0 \leqslant k \leqslant n} S_{k} .
$$

Теорема 1. При $t \geqslant 1$ справедливо следующее неравенство:

$$
\frac{\left(\mathbf{E} \bar{S}_{n}^{t}\right)^{1 / t}}{\left(\mathbf{E} \bar{X}_{n}^{t}\right)^{1 / t}+\left(\mathbf{E} B_{n}^{t}\right)^{1 / t}} \leqslant c_{t}:=\inf _{\alpha>1} \varphi_{t}(\alpha)
$$

где

$$
\varphi_{t}(\alpha)=\alpha \frac{\exp \left(2 t^{-1}(\alpha(\ln \alpha-1)+1)\right)}{\left[\exp \left((t+1)^{-1}(\alpha(\ln \alpha-1)+1)\right)-1\right]^{(t+1) / t}} .
$$

Доказательство этой теоремы проводится в п. 2 усовершенствованием рассуждений из работы С.В. Нагаева [1], где, в частности, доказывалось соответствующее неравенство с другой функцией, которая, как показано ниже, заведомо больше $c_{t}$.

Пусть $S_{k}, k \geqslant 0,-$ мартингал, $\widehat{S}_{n}=\max _{1 \leqslant k \leqslant n}\left|S_{k}\right|, D_{t}=\mathbf{E}\left(\max _{1 \leqslant k \leqslant n}\left|X_{k}\right|^{t}\right)$.

Стандартным видоизменением доказательства теоремы 1 (см., например, [1]) получаем следующее утверждение.

* Центральный экономико-математический институт РАН, ул. Красикова, 47, 117418 Москва, Россия; e-mail: presman@cemi.ras.ru

1) Работа выполнена при поддержке Российского фонда фундаментальных исследований (гранты № 07-01-00541_а и 08-01-90252-Узб_а). 